\title{
How Brain Response and eating Habits modulate Food Energy Estimation
}

Mengotti, P. ${ }^{1,2^{*}}$, Aiello, M. ${ }^{1}$, Terenzi, D. ${ }^{1}$, Miniussi, C. ${ }^{3,4}$, \& Rumiati, R.I. ${ }^{1,5}$

${ }^{1}$ Area of Neuroscience, SISSA, Via Bonomea 265, 34136 Trieste, Italy

${ }^{2}$ Cognitive Neuroscience, Institute of Neuroscience \& Medicine (INM-3), Research Centre Juelich, Leo-Brandt-Str. 5, 52425 Juelich, Germany

${ }^{3}$ Center for Mind/Brain Sciences CIMeC, University of Trento, Corso Bettini 31, 38068 Rovereto (TN), Italy

${ }^{4}$ Cognitive Neuroscience Section, IRCCS Centro San Giovanni di Dio

Fatebenefratelli, Via Pilastroni 4, 25125 Brescia, Italy

${ }^{5}$ ANVUR, Via Ippolito Nievo 35, 00153 Rome, Italy

\section{*Corresponding author:}

Dr. Paola Mengotti

Cognitive Neuroscience, Institute of Neuroscience and Medicine (INM-3)

Research Centre Juelich

Leo-Brandt-Str. 5, 52425 Juelich, Germany

phone: +49-2461-61-4686, fax: +49-2461-61-1518

email: p.mengotti@fz-juelich.de 


\begin{abstract}
The estimates we do of the energy content of different foods tend to be inaccurate, depending on several factors. The elements influencing such evaluation are related to the differences in the portion size of the foods shown, their energy density (kcal/gram), but also to individual differences of the estimators, such as their body-mass index (BMI) or eating habits. Within this context the contribution of brain regions involved in food-related decisions to the energy estimation process is still poorly understood. Here, normal-weight and overweight/obese women with restrained or non-restrained eating habits, received anodal transcranial direct current stimulation (AtDCS) to modulate the activity of the left dorsolateral prefrontal cortex (dlPFC) while they performed a food energy estimation task. Participants were asked to judge the energy content of food images, unaware that all foods, for the quantity presented, shared the same energy content. Results showed that food energy density was a reliable predictor of their energy content estimates, suggesting that participants relied on their knowledge about the food energy density as a proxy for estimating food energy content. The neuromodulation of the dlPFC interacted with individual differences in restrained eating, increasing the precision of the energy content estimates in participants with higher scores in the restrained eating scale. Our study highlights the importance of eating habits, such as restrained eating, in modulating the activity of the left dlPFC during food appraisal.
\end{abstract}

Keywords: tDCS, neuromodulation, dorsolateral prefrontal cortex, energy density, restrained eating. 


\section{Introduction}

When we consider the same amount of food, for different food types the energy content will change based on their energy density (i.e., the amount of calories per gram). Therefore, it is conceivable that our knowledge concerning the energy density of different foods is used to estimate the actual energy content of different food portions. However, people are generally imprecise in estimating the energy content of foods $[1,2,3,4,5,6,7]$. This inaccuracy seems to be due mainly to a difficulty in explicitly estimating the energy content of the actual amount of food 'on the plate', in other words the portion size $[6,7]$. Many studies showed that both changes in portion size and in energy density of foods contribute to modulate the amount of energy intake in adults and children, and the contribution of these two factors is independent and additive $[8,9,10,11]$. However, recent evidence suggests that our brain is able to track the "ideal" portion of the meal [12]. It is therefore of great interest also to determine how these two factors contribute to the estimation of the energy content of different foods.

Given the lack of neuroimaging and neuromodulation studies investigating food energy estimation, the contribution of different brain regions in this cognitive process is poorly understood. One region that was found to be widely involved in food-related decisions is the dorsolateral prefrontal cortex (dlPFC)

$[13,14,15,16,17,18,19]$. The dlPFC is generally involved in cognitive control, integrating multiple sources of information and exerting a top-down control of behavior [20]. In the food domain, the dlPFC is involved in integrating more abstract or long-term attributes of foods (i.e. the information about the level of healthiness of the food) into the choice of which food participants wanted to eat at the end of the experiment [16]. In addition, this region is involved in self-control towards food [15], 
and in the regulation of cravings $[13,14,17,18,19]$. It is hypothesized that the dlPFC may have a role in inhibiting the desire towards high-energy or unhealthy food by exerting a modulation over reward-sensitive regions, such as the medial prefrontal cortex or the orbitofrontal cortex $[16,18,19]$. The process of energy estimation is complex and requires the integration of different pieces of information, concerning the energy density of the food considered and the amount of food shown; thus the dlPFC is a good candidate to support this process. In addition, neuroimaging studies have shown that the activity of the dIPFC is altered both in overweight people and people reporting restrained eating $[21,22,23]$. Previous studies showed increased dlPFC activity in people who showed restraint eating [15,22,23], triggering cognitive control or inhibitory mechanisms when processing food. However, it is still an open question whether higher activation of cognitive control mechanisms translates into more precise food energy estimates. It is therefore interesting to explore the impact of individual differences in the process of energy estimation and in the modulation of the dlPFC activity during this cognitive process.

In the present study, overweight/obese and normal-weight participants, who differed also in their degree of restrained eating, received anodal transcranial direct current stimulation (AtDCS) and sham stimulation in order to modulate the activity of the dlPFC while they performed a food energy estimation task. Participants evaluated food pictures that shared the same energy content (i.e., $200 \mathrm{kcal}$ ), but differed in their energy densities and, as such, in the size of their portions. For instance, in order for the two portions of cucumber and chocolate to have the same energy content, the former needs to be larger than the latter, as they two have different energy densities. To provide a precise estimate of the calorie content, participants should integrate the information concerning the energy density of the food with the presented portion size. 
However, if participants' estimates are based on the energy density of foods, it would suggest a difficulty in integrating the information concerning portion size into the energy estimate. Moreover, we hypothesized that, if the dIPFC has a role in supporting the process of energy estimation, we should see that participants increased their ability in estimating the energy content of foods during AtDCS. In fact, this area is expected to be involved in integrating the information concerning the portion size shown with the previous knowledge about the food in terms of energy density. Additionally, we explored the role of individual differences in body-mass index (BMI) and restrained eating in modulating their accuracy in the energy estimation task and the involvement of the dlPFC in this process.

\section{Materials and methods}

\subsection{Participants}

Thirty-seven healthy young females participated in the study. Three of them were excluded because they showed a profile compatible with moderate depression, tested with the Beck Depression Inventory (BDI-II, [24]), three participants were excluded for abnormal food-related behavior, tested with the Eating Disorder Inventory (EDI-3, [25]), and one participant was excluded because she used psychotropic substances.

Therefore, the final sample included 30 participants (see Table 1 for participants' characteristics). Twenty-seven participants were right-handed and three were ambidextrous [26]. None of the participants had a history of neurological or other relevant medical disease, or were under pharmacological treatment that may affect cognitive performance at the time of the experiment. They had normal or corrected-to-normal vision and they did not suffer from daltonism or achromatism. They also did not have specific food restrictions such as vegetarianism or avoidance 
of specific foods because of religion, allergy or medical conditions. Participants were weighted and measured in their height at the beginning of the experimental session in order to calculate their body-mass index (BMI, in $\mathrm{kg} / \mathrm{m}^{2}$ ), used as an indicator of human body fat [27]. Fifteen participants were overweight or obese (BMI $\geq 25)$ and 15 participants were normal-weight (BMI from 18.5 to 24.9). Moreover, 19 participants reported to be restrained-eaters, therefore actively trying to restrict or control their dietary intake, whereas 11 participants reported to be non-restrainers, according to the Restraint eating scale-revised (RS-R, [28]; restrained-eaters cut-off score $=13$ ). The RS-R is a ten-item questionnaire used for measuring dietary restraints. Its items are rated on a four- to five-point scale, with a maximum total score of 35. The scale consists of two subscales: weight fluctuation (WF) and concern with dieting (CD). An Italian version of the RS-R had not been yet provided, therefore we used a translated version of the questionnaire.

All participants gave written informed consent. The study has been approved by the SISSA Ethics Committee and has been performed in accordance with the ethical standards as laid down in the Declaration of Helsinki and its later amendments. 
Table 1. Participants' characteristics

\begin{tabular}{l|c}
\hline Characteristic & Value \\
\hline Age (years) & $24.1 \pm 3.4$ \\
Education (years) & $15.1 \pm 2.1$ \\
Height $(\mathrm{cm})$ & $164 \pm 6.4$ \\
Weight $(\mathrm{kg})$ & $70.6 \pm 14$ \\
BMI $\left(\mathrm{kg} / \mathrm{m}^{2}\right)$ & $26.5 \pm 6.2$ \\
RS & $13.4 \pm 4.1$ \\
\hline
\end{tabular}

All values are reported as mean \pm SD. BMI: body-mass index; RS: restraint eating scale score.

\subsection{Stimuli and experimental paradigm}

In the main experiment, participants judged 120 pictures of foods ranging from low to high energy density (kcal/gram; food energy density range: 0.12-6.91 kcal/gram) items. The final 120 food images were selected from a pool of 159 images through a rating performed on 35 healthy participants between 18 and 35 years old (mean age: $26 \pm 3$ (SD) years; 20 females). The participants enrolled in the pre-selection of the stimuli did not participated in the main experiment. They were asked to write the name of each food shown, to rate the prototypicality of each of the food items on a Likert scale ranging from 1 to 5 points, and to rate on the Likert scale also their eating frequency of each of the foods. Thirty-nine images were excluded because either the naming responses were not consistent across participants or the degree of prototypicality was low.

The final 120 foods were presented each in a portion of $200 \mathrm{kcal}$ per picture, therefore varying the quantity of food presented depending on the energy density of each food. It is important to note that the portion size of each food shown was adapted 
in order to present food items containing always $200 \mathrm{kcal}$ per picture and only one portion size for each food was shown. Food pictures were taken from the website: http://www.caloriegallery.com.

On every trial, participants saw a fixation cross for $500 \mathrm{~ms}$ followed by a picture of one of the food items. Each picture was presented for $2000 \mathrm{~ms}$, and then a question appeared on the screen asking them to judge the energy content of the food ("How energy-heavy do you think this food is?") together with a visual analogue scale with the words "very low" and "very high" at the two extremities. The visual analogue ranged from -460 to 460 pixels in the screen, corresponding to -122 and $+122 \mathrm{~mm}$. For the analysis and the presentation of the results scale was then converted in units from -50 to +50 , each unit corresponding to $2.4 \mathrm{~mm}$ or 1.2 pixels. We maintained the positive/negative values, as they convey the idea of under- and over-estimation of the energy content. Numerical labels at the extremities were intentionally avoided, as the actual energy content of the items was always constant, i.e., $200 \mathrm{kcal}$. Participants had to move a slider from the center of the scale to give their response. They were explicitly instructed to estimate the energy content of the food in the portion presented to them. Since all food pictures share the same energy content, the optimal behavior would be to evaluate all food items as equal. To achieve this, participants should multiply the portion size with the energy density: energy content $(\mathrm{kcal})=$ portion size $($ grams $) \mathrm{x}$ energy density $(\mathrm{kcal} / \mathrm{gram})$. Therefore in the present experiment, if participants base their energy estimates on the energy density, it implies that they failed to take into account the information concerning the portion size shown.

They had no maximum time for their response. The mean duration of the task was 9 mins. The order of the stimuli was randomized. 
In addition, before the main task participants performed an assessment of their psychophysical state that included five questions pertaining to participants' current level of hunger, thirst, and tiredness, and the time they consumed the last snack and the last complete meal [29]. Participants responded by indicating a position along a visual analogue scale (ranging from -460 to 460 pixels in the screen), with end-points reflecting two extremities. No differences were found between sham and AtDCS concerning the items of the psychophysical test (paired t-tests, all $p s>0.3$ )

Mood was assessed before and after tDCS with a visual analogue scale (ranging from -460 to 460 pixels in the screen) that, with end-points reflecting two extremities, tested 16 different domains of mood: calm/restless; alert/drowsy; apathetic/dynamic; confused/lucid; strong/weak; sharp/blunt; satisfied/unfulfilled; worried/unconcerned; fast mind/slow mind; tense/relaxed; attentive/neglectful; inept/competent; happy/sad; hostile/friendly; interested/ indifferent; quiet/sociable. Participants' mood [13] was assessed in order to check for unspecific changes in mood due the neuromodulation, as the left dlPFC is frequently used as stimulation target in studies for the treatment of depression (see [30] for a review). Mood assessment was collected in 26 out of 30 participants, four participants could not complete the mood assessment due to technical problems.

Participants performed two counterbalanced sessions one week apart, one with active tDCS and one with sham (placebo) stimulation. At the end of the second session, they also completed a questionnaire that included questions to assess general information on current health and dietary habits. 


\section{3 tDCS protocol}

During the data acquisition, tDCS (anodal or sham) was administered with a batterydriven DC stimulator (Eldith, NeuroConn) using an electrode with an area of $25 \mathrm{~cm}^{2}$. The electrode was positioned over the left dlPFC, moving $8 \mathrm{~cm}$ frontally and $6 \mathrm{~cm}$ laterally from $\mathrm{Cz}$ (in 10-20 nomenclature for EEG electrode positioning, also referred as vertex). The return electrode, with an area of $70 \mathrm{~cm}^{2}$, was placed extra-cephalically on the right upper arm. We used an extra-cephalic location to avoid interference effects from brain areas beneath the return electrode. Positions like supraorbital or similar can affect the prefrontal cortex and can results in relatively widespread stimulation of brain regions (see for instance [31]). Therefore the extra-cephalic montage was chosen in order to isolate the effect of the current flow direction, as much as possible, to the left dlPFC [32,33]. In the AtDCS, the current intensity of 1.5 $\mathrm{mA}$ (current density of $0.06 \mathrm{~mA} / \mathrm{cm} 2$ ) was delivered for $720 \mathrm{~s}$, including two ramping periods of $15 \mathrm{~s}$ at the beginning and at the end of the stimulation. The stimulation started $120 \mathrm{~s}$ before the beginning of the task. During the sham session, the electrodes were located in the same positions as in the active stimulations, but the current was supplied only during the first $30 \mathrm{~s}$ (15 fade-in phase and 15 fade-out phase). This procedure ensured that the participants felt the tingling sensation at the beginning and at the end of the stimulation. At the end of the experimental protocol in each session, the participants completed a questionnaire about sensations experienced during the stimulation to evaluate whether the two stimulation conditions differed in the sensations experienced [34]. No differences between the sensations experienced during the sham and AtDCS were reported, as shown by the non-parametric Wilcoxon rank sum tests (all $p s>0.2$ ). 


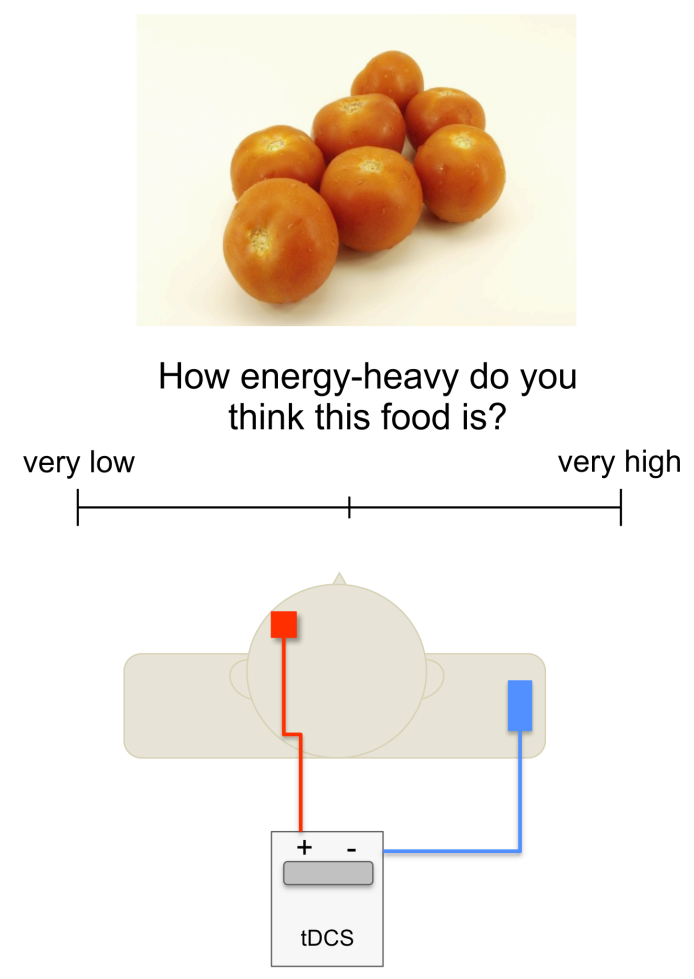

Figure 1. Experimental design. Images of different foods are presented to the participants during concurrent anodal transcranial direct current stimulation (AtDCS) over the left dorsolateral prefrontal cortex (dlPFC). Participants are requested to report how energy-heavy the food is by moving a slider over a visual analogue scale. Food image is taken from: http://freefoodpictures.caloriegallery.com.

\subsection{Statistical analyses}

We first investigated the relationship between BMI, score at the RS-R (from now on only RS) and the energy estimates during AtDCS and sham by calculating bivariate correlations between measures.

Data were then analyzed with linear mixed-effects models (LMMs) using R (version 3.3.3; http://www.r-project.org/) and in particular using the lmer function (lme4 package; http://cran.r-project.org/web/packages/lme4/index.html). The models included also a random intercept for participants and for items, to account for individual differences and variability related to the different items. 
Participants' energy estimate was the dependent variable, and a stepwise procedure was followed, adding one at a time each factor (factors: food energy density, BMI, RS, tDCS [anodal, sham]) and second-level interactions. As the actual energy content of the food items was constant to $200 \mathrm{kcal}$, this factor could not be added to the model. As BMI and RS correlated, we orthogonalized the two factors by regressing out the effect of RS from BMI and vice versa. The residual scores from the linear regression represented the effect of BMI (or of RS) without the potential confounding effect of RS (or BMI). The unstandardized residual scores entered then the LMMs. The factor food energy density was also mean centered. The degree of collinearity was tested using the condition number $k$ [35]. Models were compared using the Anova function and factors (an interactions) were kept in the model only if they caused a significant increase of fit (tested by the Akaike Information Criterion, AIC). Reduced AIC was used as criterion for model selection because it favors parsimonious models, also when the sample size is small [36]. As an additional control analysis, the winning model was contrasted against alternative models that were created by removing one at a time the significant factors via parametric bootstrapping (1000 simulations). Bootstrap 95\% confidence intervals of the winning model were calculated (1000 simulations).

\section{Results}

\subsection{Food energy estimates}

The factors BMI and RS showed were positively correlated $(\mathrm{r}(30)=0.38, \mathrm{p}=0.04)$. Therefore for these two factors we entered in the correlation analysis and in the linear mixed models analysis unconfounded values, obtained by removing from the BMI values putative linear effects of RS. The same procedure was followed for RS values. 
RS correlated with the energy estimate scores during sham $(\mathrm{r}(30)=0.4, \mathrm{p}=$ 0.03), but not with the energy estimate scores during AtDCS $(\mathrm{r}(30)=0.19, \mathrm{p}=0.3)$. No significant correlations were found between BMI and energy estimates.

Table 2. Results of the correlational analysis.

\begin{tabular}{|c|c|c|c|}
\hline & & Energy estimates & Energy estimates \\
\hline & BMI & AtDCS & sham \\
\hline RS & $0.38 *$ & & \\
\hline RS (residuals) & & 0.19 & $0.4^{*}$ \\
\hline BMI (residuals) & & -0.28 & -0.32 \\
\hline
\end{tabular}

As for the linear mixed models analysis, the best fitting model showed that participants' energy estimates mainly depended on the effect of food energy density, on the interaction between RS and tDCS, and on the interactions of food energy density with BMI and food energy density with RS. See Table 3 for descriptive data of single parameters and significance. The condition number $k$ assessing the degree of collinearity was 2.8 , indicating reasonable collinearity $(<10)$. The winning model had also a better fit (using the parametric bootstrapping) than alternative models created by removing the significant interaction tDCS and RS $(\mathrm{p}=$ 0.001), by removing the significant interaction food energy density and RS ( $p=$ $0.001)$, and by removing the significant interaction food energy density and BMI ( $\mathrm{p}=$ $0.001)$. 
Table 3. Summary of the best fitting LMM for food energy estimates.

\begin{tabular}{|c|c|c|c|c|c|c|}
\hline \multirow{2}{*}{$\begin{array}{l}\text { Energy estimates } \\
\text { Fixed effects }\end{array}$} & \multirow[b]{2}{*}{$\beta$} & \multirow[b]{2}{*}{ SEM } & \multirow[b]{2}{*}{$t$} & \multirow[b]{2}{*}{$p$} & \multicolumn{2}{|c|}{$95 \% \mathrm{CI}$} \\
\hline & & & & & Lower & Upper \\
\hline Intercept & 1.84 & 1.6 & 1.2 & 0.24 & -1.13 & 4.88 \\
\hline Energy density & 8.79 & 0.69 & 12.7 & $<0.001$ & 7.35 & 10.31 \\
\hline BMI & -0.23 & 0.18 & -1.2 & 0.22 & -0.58 & 0.12 \\
\hline $\mathrm{RS}$ & 0.44 & 0.28 & 1.6 & 0.13 & -0.15 & 0.99 \\
\hline $\mathrm{tDCS}(A t D C S)$ & -0.71 & 0.38 & -1.9 & 0.06 & -1.48 & 0.08 \\
\hline $\mathrm{RS} \times \mathrm{tDCS}(A t D C S)$ & -0.25 & 0.1 & -2.5 & 0.01 & -0.46 & -0.06 \\
\hline Energy density x BMI & -0.11 & 0.02 & -5.7 & $<0.001$ & -0.15 & -0.08 \\
\hline Energy density $x$ RS & -0.13 & 0.03 & -4.1 & $<0.001$ & -0.18 & -0.06 \\
\hline
\end{tabular}

Random effects

Subjects (intercept)

Items (intercept)

Residual

$\mathrm{AIC}=60981$
Std.Dev.

13.4

16.1

$\beta$ : beta estimate; SEM: standard error; $95 \% \mathrm{CI}$ : bootstrap confidence interval; BMI: body-mass index; RS: restraint score; AtDCS: anodal tDCS; AIC: Akaike Information Criterion. Significant $p$ values are in bold. Reference condition for the categorical factor is reported in italic in brackets.

The main effect of food energy density suggests that participants estimated the energy content of food relying on their knowledge concerning the energy density of foods shown, leading to an overestimation of the energy content of high-energy dense 
foods (response values higher than zero) and to an underestimation of the energy content of low-energy dense foods (response values lower than zero).

Concerning the effect of tDCS over energy estimates, we found an interaction between RS and tDCS effect. During the sham condition, participants with lower scores in RS tended to slightly underestimate the energy content, whereas participants with higher scores in RS tended to overestimate the energy content of the foods. When AtDCS was applied, we found a decrease in the energy estimates of participants with high RS scores, leading therefore to more precise estimates (see Figure 2).

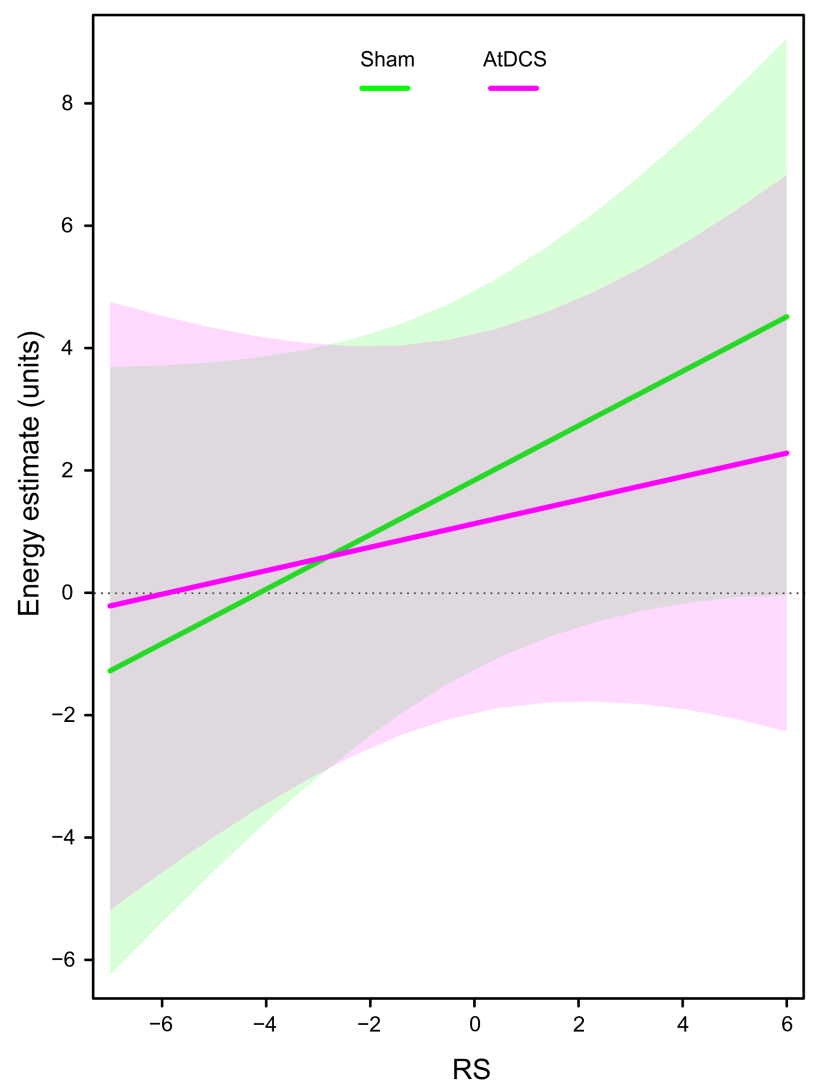

Figure 2. Effect of tDCS on energy estimates. tDCS x RS interaction: energy estimates get more precise during AtDCS, in particular by decreasing energy estimates of participants with high RS. 
In addition, participants' BMI modulated the effect of food energy density: especially for high-energy dense foods, the higher participants' BMI the less pronounced was the overestimation of the actual energy content of those foods, leading to more precise estimates of actual energy content (see Figure 3A). Also participants' RS modulated the effect of food energy density: especially for lowenergy dense foods, the higher participants' RS the less pronounced was the underestimation of the actual energy content of those foods, leading to more precise estimates of actual energy content (see Figure 3B).

A

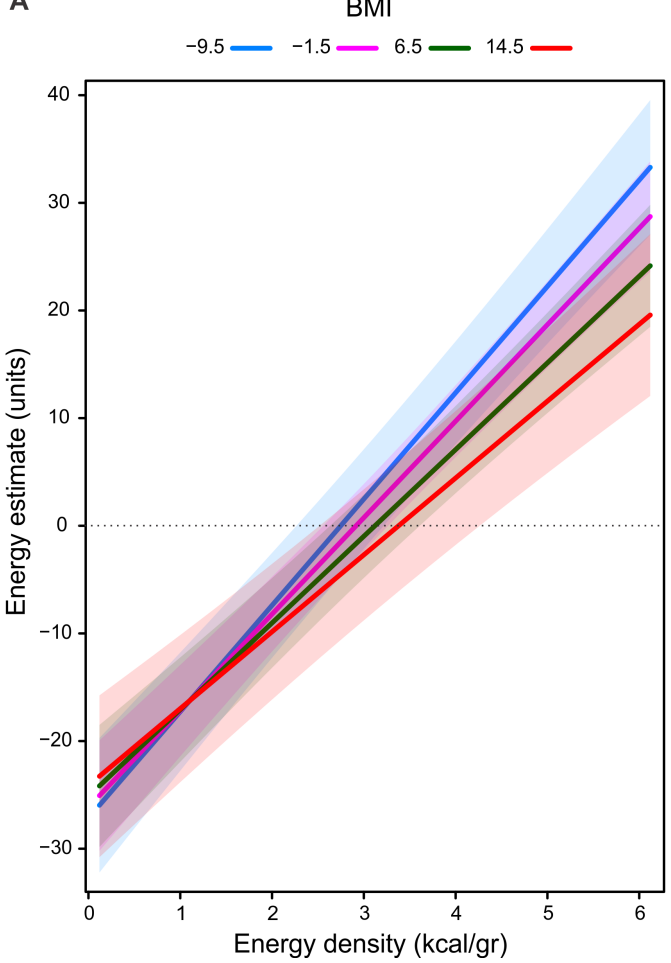

B

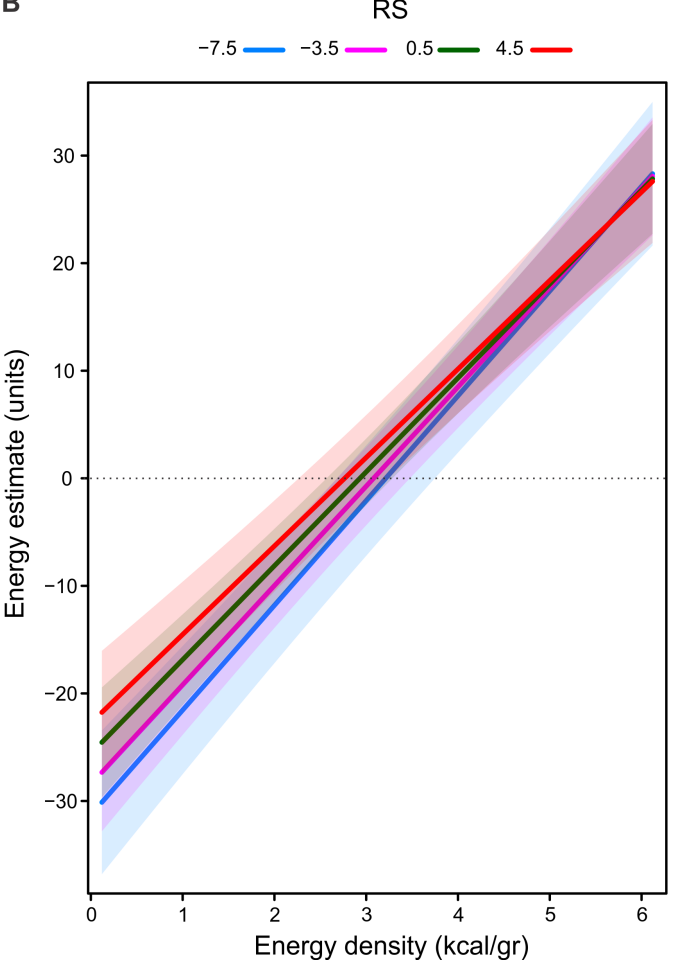

Figure 3. Effect of energy density on energy estimates. A) Energy density x BMI interaction: energy estimates get more precise with increasing BMI; B) Energy density x RS interaction: energy estimates get more precise with increasing RS. 


\subsection{Mood assessment}

To assess that our results were not confounded by unspecific changes in mood due to the AtDCS, we tested for differences in pre- and post-stimulation change for sham and AtDCS in the 16 different domains of the mood assessment. No significant changes in mood were found between sham and AtDCS (all ps>0.05). The results of the paired t-test are shown in the Supplementary materials in Table S1.

\section{Discussion}

In the present study, we applied AtDCS over the left dlPFC aiming at modulating participants' ability to estimate food energy content. We employed a food energy estimation task specifically designed to identify the factors that may influence participants' performance. Moreover, we inspected whether participants' individual differences concerning BMI or self-reported restrictive dietary habits (RS) could impact on the modulation of the behavior induced by AtDCS or on the energy estimates.

We showed that the energy density of foods was a reliable predictor of participants' energy estimates of actual energy content of the food portion shown (in interaction with BMI and RS). Given that the actual energy content was constant for all food items, this suggests that, even if participants were explicitly instructed to judge the food energy content based on the amount presented, their responses were based on the food energy density. Therefore, energy density is creating a systematic imprecision in energy estimation, with underestimation of low-energy foods and overestimation of high-energy foods. This result is in line with the previous evidence [2,3] that people tend to overestimate the energy content of high-energy foods (perceived as unhealthy) and under-estimate the energy content of low-energy foods (perceived 
as healthy). In addition, Foroni et al. [29] reported a significant positive correlation between estimates of the energy density of different foods and their actual energy density. This latter evidence together with present data suggests that we are informed concerning the energy density of different foods, but this knowledge is not adapted to the portion size shown.

As for the effect of the modulation of the energy estimates by AtDCS over the left dlPFC, results showed that the tDCS effect was modulated by participants' RS. When participants received sham stimulation, the energy estimates tended to increase with increasing RS. In particular, participants with high RS tended to overestimate the energy content of foods. However, when the same participants underwent AtDCS, their energy estimates became more precise, especially by decreasing the energy estimates in participants with higher RS. Previous studies showed increased dlPFC activity in people who showed restraint eating during choices involving healthy and unhealthy foods [15], during food consumption [22] and in tasks requiring inhibition towards food [23]. In our study restrained eaters showed the tendency to overestimate the energy content of food items, and the anodal stimulation of the dlPFC, supposedly facilitating the depolarization of the underlying neurons, increased the precision of their estimates. Differently from previous studies, here we only required participants to give energy estimates of different types of food; as such, our paradigm does not require participants to inhibit responses towards food or food consumption. We hypothesized that, compared with lower RS participants, participants with higher RS do not show increased activation of the dlPFC in performing this task. In addition, our result may suggest that restraint eaters might be more responsive to the neuromodulation of the activity of the dlPFC during energy estimation. More generally, the AtDCS effect depends on the level of ongoing activity of the stimulated 
area/network [37]. We can speculate that the activity of the left dlPFC during our food energy estimation task is at different baseline levels in participant with high or low score at the RS. However our results cannot be considered as conclusive, as previous neuroimaging studies did not focus specifically on the process of energy estimation.

Interestingly, although BMI had an effect on energy estimations by interacting with food energy density, this factor did not interact with the tDCS. This result is informative for future research, suggesting that neuromodulation with the aim of training or improving energy estimates should take into account the degree of selfreported restrained eating and not simply volunteers' BMI.

Concerning the type of neuromodulation applied in the present study, our rationale to apply AtDCS over the left dlPFC was inspired by previous fMRI studies showing activation of the left dlPFC in response to high-energy dense foods $[16,38]$ and by studies showing alterations in the activity of this region both for overweight people and restraint eaters $[21,22,23]$. Previous studies in which neuromodulation was applied over the dIPFC to reduce cravings $[13,14,18]$ used mainly a bilateral tDCS montage, and their results point towards an effective role of the cathodal stimulation over the left dlPFC with concurrent anodal stimulation over the right dlPFC. However, one other study showed decreased cravings when the activity of left dlPFC was increased through high-frequency TMS [19].

In addition, we found that individual differences such as BMI and RS interacted with the factor energy density to predict participants' energy estimates. The interaction between energy density and BMI indicated that the higher the BMI, the more precise tended to be the energy estimate. Estimates of the actual energy content "erroneously" increased with increasing energy density of foods, but this effect was less pronounced in participants with higher BMI, suggesting that people with higher 
BMI are actually more accurate in their estimates. The effect was particularly visible for high-energy dense foods, with a decrease in the overestimation of the energy content with increasing BMI. It is worth remembering that in this experiment as all food items share the same energy content, energy estimates should have not increased with increasing energy density of foods. This result is in contrast with previous evidence suggesting higher imprecision of obese individuals in estimation of food energy content [1], and with a meta-analysis showing that people with high BMI are more prone to underestimate the energy content of their meals [5]. However, one other study showed that the association between BMI and underestimation of meal energy content could be due not to the difference in BMI itself, but more simply to the tendency of people with higher BMI to consume larger meals [6]. A similar effect was found for the interaction between energy density and restraint eating, the higher was the RS the more precise tended to be the energy estimates. Estimates of the actual energy content "erroneously" increased with increasing energy density of foods, but this effect was less pronounced in participants with higher RS. In particular, participants with a lower RS tended to underestimate the energy content of lowenergy dense foods, and this effect was less noticeable in participants with high RS. This is in line with previous evidence showing that dieters were more accurate than non-dieters in energy estimation of healthy foods, but both groups were grossly inaccurate in their estimation, overestimating the energy content of high-energy foods and underestimating the energy content of low-energy foods [3]. However, there is also evidence that participants who overestimate the energy content of foods are more likely to report restrained eating [39]. In addition, it is not clear whether restrained eating is a useful behavior for weight loss or maintenance. In fact, even if restrained eaters tend to overestimate the energy content of foods [39], they do not eat less than 
non-restrained eaters $[40,41]$. Moreover, dieting is also a predictor of weight gain in normal-weight people [42], indicating that restrained eating might not be an effective strategy for weight control. Restrained eating was also shown to predict BMI, the higher the level of restrained eating the higher the BMI [43]. It should be specified that our RS is based on self-report, and probably is a reflection of the attempts to restrict energy intake, whether successfully or not.

One limitation of our study is represented by our sample size $(n=30)$, that might be considered relatively small for the complexity of the model applied to the data. To ensure the reliability of our results we performed further model comparisons via parametric bootstrapping, as described in the Results. Furthermore, we analyzed the data using two separate models (data not shown), one testing for the effects of energy density, BMI and tDCS, and the other testing for the effects of energy density, RS and tDCS. If the results of the two separate (and simpler) models showed similar effects to the results of our comprehensive model, it would represent strong evidence in favour of our results. The results of this analysis were not different from those of our comprehensive model, further corroborating the validity of the reported results. Nevertheless, further investigations using neuroimaging to evaluate the process of energy estimation are needed to clarify the present results.

A second possible limitation is the translational value of our results. The main characteristic of our study design is that all foods share a fixed energy content of 200 kcal, while in daily life, meals do not have the same amount of energy content and usually foods with higher energy density are also associated with higher energy content. Therefore, the heuristic of associating a higher energy content to high energy density foods might be useful in many situations in which individuals need to make feeding decisions. However, as previously described, as portion size together with 
food energy density modulate energy intake $[8,9,10,11]$, it is important to understand how these two factors interact to determine energy estimates.

In conclusion, our data provide new important information concerning the role of the left dlPFC in food energy estimation. We showed that the neuromodulation of the dlPFC interacts with individual differences with respect to restrained eating to modulate the process of energy estimation. We provided also new evidence concerning how humans estimate food energy content in relation to individual differences, and which are the factors that contribute to this process.

\section{Acknowledgements}

This work was supported by Beneficentia. We are grateful to C. Cecchetto for her help with linear mixed models. We thank also S. Viswanathan for helpful comments and suggestions on an earlier version of the manuscript.

\section{Disclosure of interest.}

The authors report no conflicts of interest. 


\section{References}

[1] L.G. Bandini, D.A. Schoeller, H.N. Cyr, W.H. Dietz, Validity of reported energy intake in obese and nonobese adolescents, Am. J. Clin. Nutr. 52(3) (1990) 421425.

[2] R.A. Carels, J. Harper, K. Konrad, Qualitative perceptions and caloric estimations of healthy and unhealthy foods by behavioral weight loss participants, Appetite 46(2) (2006) 199-206.

[3] R.A. Carels, K. Konrad, J. Harper, Individual differences in food perceptions and calorie estimation: An examination of dieting status, weight, and gender, Appetite 49(2) (2007) 450-458.

[4] S.W. Lichtman, K. Pisarska, E.R. Berman, M. Pestone, H. Dowling, E. Offenbacher, et al., Discrepancy between self-reported and actual caloric intake and exercise in obese subjects, N. Engl. J. Med. 327(27) (1992) 1893-1898.

[5] M.B.E. Livingstone, A.E. Black, Biomarkers of nutritional exposure and nutritional status, J. Nutr. 133(3) (2003) 895-920.

[6] B. Wansink, P. Chandon, Meal size, not body size, explains errors in estimating the calorie content of meals, Ann. Intern. Med. 145(5) (2006) 326-332.

[7] C.K. Martin, S.D. Anton, E. York-Crowe, L.K. Heilbronn, C. VanSkiver, L.M. Redman, et al., Empirical evaluation of the ability to learn a calorie counting system and estimate portion size and food intake, Br. J. Nutr. 98(02) (2007) $439-444$.

[8] J.O. Fisher, Y. Liu, L.L. Birch, B.J. Rolls, Effects of portion size and energy density on young children's intake at a meal, Am. J. Clin. Nutr. 86(1) (2007) 174-179. 
[9] S.M. Kling, L.S. Roe, K.L. Keller, B.J. Rolls, Double trouble: Portion size and energy density combine to increase preschool children's lunch intake, Physiol. Behav. 162 (2016) 18-26.

[10] T.V. Kral, L.S. Roe, B.J. Rolls, Combined effects of energy density and portion size on energy intake in women, Am. J. Clin. Nutr. 79(6) (2004) 962-968.

[11] B.J. Rolls, E.L. Morris, L.S. Roe, Portion size of food affects energy intake in normal-weight and overweight men and women, Am. J. Clin. Nutr. 76(6) (2002) 1207-1213.

[12] U. Toepel, M.L. Bielser, C. Forde, N. Martin, A. Voirin, J. le Coutre, et al., Brain dynamics of meal size selection in humans, NeuroImage 113 (2015) 133-142.

[13] F. Fregni, F. Orsati, W. Pedrosa, S. Fecteau, F.A.M. Tome, M.A. Nitsche, et al., Transcranial direct current stimulation of the prefrontal cortex modulates the desire for specific foods, Appetite 51(1) (2008) 34-41.

[14] R.L. Goldman, J.J. Borckardt, H.A. Frohman, P.M. O’Neil, A. Madan, L.K. Campbell, et al., Prefrontal cortex transcranial direct current stimulation (tDCS) temporarily reduces food cravings and increases the self-reported ability to resist food in adults with frequent food craving, Appetite 56(3) (2011) 741-746.

[15] T.A. Hare, C. Camerer, A. Rangel, Self-control in decision-making involves modulation of the vmPFC valuation system, Science 324 (2009) 646-648.

[16] T.A. Hare, J. Malmaud, A. Rangel, Focusing attention on the health aspects of foods changes value signals in vmPFC and improves dietary choice, $\mathrm{J}$. Neurosci. 31(30) (2011) 11077-87.

[17] C.A. Hutcherson, H. Plassmann, J.J. Gross, A. Rangel, Cognitive regulation during decision making shifts behavioral control between ventromedial and dorsolateral prefrontal value systems. J. Neurosci. 32(39) (2012) 13543-13554. 
[18] M. Kekic, J. McClelland, I.C. Campbell, S. Nestler, K. Rubia, A.S. David, et al., The effects of prefrontal cortex transcranial direct current stimulation (tDCS) on food craving and temporal discounting in women with frequent food cravings, Appetite 78 (2014) 55-62.

[19] R. Uher, D. Yoganathan, A. Mogg, S.V. Eranti, J. Treasure, I.C. Campbell, et al., Effect of left prefrontal repetitive transcranial magnetic stimulation on food craving, Biol. Psychiatry 58(10) (2005) 840-842.

[20] E.K. Miller, J.D. Cohen, An integrative theory of prefrontal cortex function, Annu. Rev. Neurosci. 24(1) (2001) 167-202.

[21] L. Batterink, S. Yokum, E. Stice, Body mass correlates inversely with inhibitory control in response to food among adolescent girls: An fMRI study, NeuroImage 52(4) (2010) 1696-1703.

[22] K.S. Burger, E. Stice, Relation of dietary restraint scores to activation of rewardrelated brain regions in response to food intake, anticipated intake, and food pictures, NeuroImage 55(1) (2011) 233-239.

[23] D. Dong, X. Lei, T. Jackson, Y. Wang, Y. Su, H. Chen, Altered regional homogeneity and efficient response inhibition in restrained eaters, Neuroscience 266 (2014) 116-126.

[24] A.T. Beck, R.A. Steer, G.K. Brown, Beck Depression Inventory-II (BDI-II), San Antonio, TX: Psychological Corporation, 1996.

[25] D.M. Garner, M.P. Olmstead, J. Polivy, Development and validation of a multidimensional eating disorder inventory for anorexia-nervosa and bulimia, Int. J. Eat. Disord. 2(2) (1983) 15-34.

[26] R.C. Oldfield, The assessment and analysis of handedness: the Edinburgh inventory, Neuropsychologia 9(1) (1971) 97-113. 
[27] A. Keys, F. Fidanza, M.J. Karvonen, N. Kimura, H.L. Taylor, Indices of relative weight and obesity, J. Chronic Dis. 25(6) (1972) 329-343.

[28] C.P. Herman, J. Polivy, Restrained eating, in A. Stunkard (Ed.), Obesity, Philadelphia, PA: Saunders, 1980, pp. 208-225.

[29] F. Foroni, G. Pergola, G. Argiris, R.I. Rumiati, The FoodCast research image database (FRIDa), Front. Hum. Neurosci. 7:51 (2013).

[30] U. Palm, A. Hasan, W. Strube, F. Padberg, tDCS for the treatment of depression: a comprehensive review, Eur. Arch. Psychiatry Clin. Neurosci. 266(8) (2016) 681-694.

[31] A.R. Brunoni, M.A. Nitsche, N. Bolognini, M. Bikson, T. Wagner, L. Merabet, et al., Clinical research with transcranial direct current stimulation (tDCS): challenges and future directions, Brain Stimul. 5 (2012) 175-95.

[32] A.Y. Kabakov, P.A. Muller, A. Pascual-Leone, F.E. Jensen, A. Rotenberg, Contribution of axonal orientation to pathway-dependent modulation of excitatory transmission by direct current stimulation in isolated rat hippocampus, J. Neurophysiol. 107 (2012) 1881-9.

[33] G.M. Noetscher, J. Yanamadala, S.N. Makarov, A. Pascual-Leone, Comparison of cephalic and extracephalic montages for transcranial direct current stimulation-a numerical study, IEEE Trans. Biomed. Eng. 61(9) (2014) 24882498.

[34] A. Fertonani, C. Ferrari, C. Miniussi, What do you feel if I apply transcranial electric stimulation? Safety, sensations and secondary induced effects, Clin. Neurophysiol. 126(11) (2015) 2181-2188.

[35] D. Belsley, E. Kuth, R. Welsch, Regression Diagnostics. Indentifying influential data and sources of collinearity, Wiley, New York, 1980. 
[36] B.M. Bolker, M.E. Brooks, C.J. Clark, S.W. Geange, J.R. Poulsen, M.H.H. Stevens, J.S.S. White, Generalized linear mixed models: A practical guide for ecology and evolution, Trends Ecol. Evol. 24 (2009) 127-135.

[37] A. Fertonani, C. Miniussi, Transcranial Electrical Stimulation: What we know and do not know about mechanisms, Neuroscientist, 23(2) (2017) 109-123.

[38] W.D. Killgore, A.D. Young, L.A. Femia, P. Bogorodzki, J. Rogowska, D.A. Yurgelun-Todd, Cortical and limbic activation during viewing of high-versus low-calorie foods, NeuroImage, 19(4) (2003) 1381-1394.

[39] A.L. Stanton, T.A. Tips, Accuracy of calorie estimation by females as a function of eating habits and body mass. Int. J. Eat. Disord. 9(4) (1990) 387-393.

[40] M.L.L. Scott, S.M.M. Nowlis, N. Mandel, A.C.C. Morales, The effects of reduced food size and package size on the consumption behavior of restrained and unrestrained eaters, J. Consum. Res. 35(3) (2008) 391-405.

[41] E. Stice, R. Sysko, C.A. Roberto, S. Allison, Are dietary restraint scales valid measures of dietary restriction? Additional objective behavioral and biological data suggest not, Appetite 54(2) (2010) 331-339.

[42] M.R. Lowe, S.D. Doshi, S.N. Katterman, E.H. Feig, Dieting and restrained eating as prospective predictors of weight gain, Front. Psychol. 4: 5777 (2013).

[43] M. Price, S. Higgs, M. Lee, Self-reported eating traits: Underlying components of food responsivity and dietary restriction are positively related to BMI, Appetite 95 (2015) 203-210. 